Regards sur l'économie allemande

Bulletin économique du CIRAC

$80 \mid 2007$

Varia

\title{
Politique d'immigration : de nouvelles orientations
}

\section{Werner Zettelmeier}

\section{OpenEdition}

\section{Journals}

Édition électronique

URL : http://journals.openedition.org/rea/706

DOI : $10.4000 /$ rea. 706

ISBN : 978-2-8218-0856-0

ISSN : 1965-0787

Éditeur

CIRAC

Édition imprimée

Date de publication : 1 mars 2007

Pagination : $35-36$

ISSN : 1156-8992

Référence électronique

Werner Zettelmeier, « Politique d'immigration : de nouvelles orientations », Regards sur l'économie allemande [En ligne], 80 | mars 2007, document 1, mis en ligne le 01 mars 2009, consulté le 15 septembre 2020. URL : http://journals.openedition.org/rea/706

Ce document a été généré automatiquement le 15 septembre 2020.

(c) CIRAC 


\title{
Analyse \\ Politique d'immigration : de nouvelles orientations
}

\author{
Werner Zettelmeier
}

1 L'Allemagne cherche à intégrer les quelque 190000 étrangers tolérés sur son territoire en attente d'une solution durable à leur situation. La plupart d'entre eux sont originaires de Turquie, de l'ex-Yougoslavie et de l'Irak, un peu moins de deux tiers vit en Allemagne depuis plus de 5 ans. Une réforme de la législation leur ouvrirait le droit d'obtenir la nationalité allemande. Cette politique d'assimilation permettrait d'apporter une réponse à une série de questions et problèmes : exclusion sociale, dérive des budgets sociaux des communes, dénatalité ou manque de main d'œuvre qualifiée. Elle s'inscrit dans le cadre d'une révision générale de la politique d'immigration allemande, fondée sur le constat que l'Allemagne est devenu de fait un pays d'immigration.

\section{Un projet de loi en négociation depuis l'été $2006 .$.}

Dans une négociation entamée après l'été 2006, le ministère fédéral du Travail (SPD) et le ministère fédéral de l'Intérieur (CDU) avaient présenté à la mi-novembre 2006 un compromis portant notamment sur la question des étrangers «tolérés» (bleibeberechtigte Ausländer). Ce texte a été par la suite soumis aux ministres de l'Intérieur des Länder, compétents en matière de droit relatif au séjour des étrangers en Allemagne, afin qu'ils puissent s'exprimer à leur tour et donner leur accord pour que le compromis des ministres fédéraux puisse déboucher sur une nouvelle loi fédérale. Celle-ci doit modifier la Loi relative à la régulation de l'immigration (Zuwanderungsgesetz) qui comprend deux volets : le premier régulant le séjour, l'activité professionnelle et l'intégration des étrangers, le second la libre circulation des citoyens européens. Cette loi, entrée en vigueur le $1^{\mathrm{er}}$ janvier 2005, a pour objectif déclaré « une régulation et organisation de l'immigration dans le respect des capacités d'accueil et 
d'intégration ainsi que des intérêts en termes économiques et de marché de l'emploi de la République fédérale ».

\section{... pour réviser la Loi sur l'immigration de $2005 .$.}

3 Cette loi, fruit d'un difficile compromis élaboré au printemps 2004 entre le gouvernement SPD-Verts de l'époque et l'opposition chrétienne-démocrate, majoritaire au Bundesrat, accepte pour la première fois la réalité de l'Allemagne comme pays d'immigration. Elle s'efforce de régler à la fois les conditions d'immigration et l'intégration des étrangers dans un même texte législatif, elle tente d'améliorer la situation des réfugiés séjournant en Allemagne, et elle ouvre par ailleurs des perspectives - limitées - pour une immigration contrôlée d'une main d'oeuvre étrangère qualifiée dont l'Allemagne aura de plus en plus besoin à l'avenir compte tenu de la faiblesse persistante de sa natalité. En effet, en 2006 tout comme dans les années qui précèdent, le nombre des décès a dépassé de loin le nombre des naissances (150 000), et le léger excédent du solde migratoire (30 000) n'a pas suffi pour éviter une légère diminution de la population totale.

\section{... qui améliorait l'intégration des étrangers}

Dans l'esprit de la loi de 2005, la priorité de l'action politique doit aller à l'amélioration de l'intégration des 6,7 millions d'étrangers résidant de façon durable en Allemagne avant de faciliter l'arrivée de nouveaux immigrants. $32 \%$ des étrangers vivant en Allemagne sont des ressortissants d'un pays de l'UE, $48 \%$ d'un pays européen autre que communautaire (y compris la Turquie avec quelque 1,7 million de personnes, soit $26 \%$, le groupe national le plus nombreux), $12 \%$ d'un pays d'Asie, $3 \%$ d'un pays d'Afrique et $3 \%$ d'un pays du continent américain, selon Destatis. A noter également que 1,4 million d'étrangers, soit $21 \%$ de tous les étrangers vivant officiellement outre-Rhin, sont nés en Allemagne. Parmi ceux-ci, les Turcs (34\%), les Italiens (30\%) et les Néerlandais $(28 \%)$ sont les plus nombreux. A ces chiffres officiels, il faut ajouter les étrangers séjournant de façon illégale ou clandestine (les « sans papiers » à l'allemande), difficiles à chiffrer par définition, mais qui sont estimés à 0,5 voire 1 million de personnes selon les sources.

\section{$20 \%$ de la population est étrangère ou issue de l'immigration}

5 La question de l'immigration constitue depuis les années 1980 un enjeu essentiel du débat politique (ouest)allemand suscitant, bien au-delà des clivages politiques traditionnels, des réactions parfois très virulentes et passionnelles au sein de l'opinion publique. Elles ont longtemps handicapé toute tentative prudente de rationalisation de la question sur fond d'une réalité pourtant de plus en plus évidente : avec actuellement un peu plus de 15 millions de personnes issues de l'immigration (rapatriés compris), dont la moitié n'a pas la nationalité allemande - soit près de $20 \%$ de la population totale ( 82 millions d'habitants), l'Allemagne est non seulement devenue de fait un pays 
d'immigration, mais aussi une société profondément métissée, comme l'a révélé l'édition 2005 du Mikrozensus présentée en juin 2006 par Destatis (voir REA 77/06).

\section{Revoir le statut précaire des étrangers « tolérés »}

6 Si la loi de 2005 constitue une étape importante dans l'adaptation indispensable de la législation allemande en matière d'immigration et d'intégration aux défis actuels, elle doit désormais être adaptée à une réglementation européenne en évolution et résoudre certains problèmes comme les modalités du rapprochement des conjoints et surtout le statut réservé aux étrangers dits "tolérés». Dans la plupart des cas, il s'agit de demandeurs d'asile qui n'ont finalement pas obtenu l'asile politique, mais dont le séjour sur le sol allemand est toléré. En effet, faute de papiers en règle émis par leur pays d'origine ou parce qu'ils risquent d'être persécutés dans leur pays d'origine, ces étrangers ne sont pas expulsés du territoire allemand. Ils vivent cependant dans une grande précarité juridique et sociale car jusqu'à présent ils n'avaient pas le droit de chercher librement un travail pour subvenir à leurs besoins et pouvaient être expulsés à tout moment si les autorités allemandes le décidaient. Ils sont quelque 190000 dans ce cas. Leur nombre est en hausse compte tenu du faible taux de reconnaissance du droit d'asile par rapport au nombre de demandeurs, même si ce dernier est en forte diminution par rapport aux années 1990 (400 000 en 1992 contre moins de 50000 en 2004). Cette situation fait l'objet d'un vif débat politique depuis une dizaine d'années.

7 Le gouvernement de grande coalition souhaite donc réformer la loi sur l'immigration de 2005. D'une part, la situation de ces étrangers tolérés ne s'est en effet nullement améliorée. D'autre part, la procédure pour faciliter l'installation de la main d'oeuvre qualifiée en Allemagne (les diplômés de l'enseignement supérieur apportant leur savoir-faire et disposant d'un revenu de $85000 €$ par an) qui était prévue dans la loi de 2005 s'est révélée peu attractive, comme l'atteste le faible nombre de candidatures. Le projet de réforme porte sur plusieurs points qui sont pour la plupart d'entre eux la conséquence de la nécessité de mettre en œuvre en Allemagne une série de directives européennes. Un aspect important de la modification concerne la régularisation des étrangers tolérés.

\section{Un parcours d'intégration pour les étrangers "tolérés »}

8 Le parcours pour régulariser définitivement leur séjour par l'obtention de la nationalité allemande serait le suivant: les personnes séjournant en Allemagne depuis au moins 6 ans (pour les familles) et 8 ans (célibataires) se voient attribuer un titre de séjour dont la durée est limitée à 2 ans. Pendant cette période, les étrangers concernés pourront exercer légalement une activité professionnelle, possibilité qui jusqu'à présent ne leur était ouverte que si aucun Allemand ou étranger disposant d'une autorisation de travail permanente ne se portait candidat au poste convoité par le demandeur d'asile. De fait, ce dernier était donc exclu du marché du travail, car cette condition était rarement réunie, d'autant plus que le demander d'asile débouté était assigné à résidence dans la ville de son séjour. Désormais il sera libre de ses mouvements sur la totalité du territoire pour chercher un travail pendant les deux ans. Pour les jeunes en particulier, 
cette disposition leur permet de commencer une formation professionnelle comme apprentis, car jusqu'à présent une place d'apprentissage était soumise à l'obtention d'une autorisation de travail que les agences locales du travail refusaient aux jeunes étrangers tolérés, même s'ils avaient de très bons résultats scolaires à l'issue de leur scolarité obligatoire. Si au bout de ces deux ans, l'étranger est en mesure d'apporter la preuve de pouvoir subvenir à ses besoins par ses propres moyens, il peut voir sa situation régularisée pour de bon: il peut obtenir la nationalité allemande si par ailleurs il passe avec succès un test de langue allemande et si son casier judiciaire est vierge. S'il n'est pas en mesure de subvenir à ses besoins, et reste par conséquent tributaire des aides sociales dont peuvent bénéficier les demandeurs d'asile, il sera 'rétrogradé' à l'état d'étranger toléré.

Si ce texte présente une avancée par rapport à la situation très précaire des demandeurs d'asile déboutés, les conditions d'application plutôt restrictives font que, selon les estimations des associations et des Eglises qui s'occupent de ces personnes, moins de 100000 étrangers tolérés (certains spécialistes évoquent un tiers seulement) pourraient bénéficier des nouvelles dispositions. De plus, le ministre de l'Intérieur de Bavière souhaite exclure tous les 9000 Irakiens tolérés de cette nouvelle disposition, car il y voit un risque pour la sécurité intérieure de la République fédérale. Si les grandes lignes des modifications relatives de ce "droit à rester en Allemagne" (Bleiberecht) semblent recueillir un consensus assez large, les modalités pratiques de mise en œuvre divisent les responsables politiques. La décision de principe adoptée par les ministres de l'Intérieur des 16 Länder le 17 novembre 2006 prévoit que les étrangers tolérés doivent d'abord chercher un emploi avant d'obtenir le titre de séjour de 2 ans. Or, certains Länder, dont la Rhénanie du Nord-Westphalie, reprochent maintenant au ministre fédéral de l'Intérieur de vouloir inscrire dans la loi le processus inverse, c'està-dire que les étrangers tolérés recevraient d'abord le titre de séjour avant qu'ils ne cherchent un travail. Le gouvernement régional reproche au Ministre fédéral de l'Intérieur de pérenniser ainsi le recours des étrangers aux aides sociales et de ne pas les encourager suffisamment à subvenir à leurs besoins par leurs propres moyens. L'entrée en vigueur de la nouvelle loi, prévue initialement pour janvier 2007, semble pour l'instant compromise.

\section{INDEX}

Mots-clés : droit d'asile, emploi, étranger, immigration, marché du travail 